\title{
sciendo
}

Int. J. of Applied Mechanics and Engineering, 2021, vol.26, No.3, pp.12-27

DOI: 10.2478/ijame-2021-0032

\section{PROBABILISTIC MESOSCALE ANALYSIS OF CONCRETE BEAMS SUBJECTED TO FLEXURE}

\author{
Haider M. AL-JELAWY*, \\ Department of Roads and Transportation, University of Al-Qadisiyah, Al Diwaniyah, IRAQ \\ E-mail: haider.aljelawy@qu.edu.iq
}

Ayad AL-RUMAITHI, Aqeel T. FADHIL and Mohannad H. AL-SHERRAWI

Department of Civil Engineering, College of Engineering, University of Baghdad, Baghdad, IRAQ

\begin{abstract}
In this paper, the probabilistic behavior of plain concrete beams subjected to flexure is studied using a continuous mesoscale model. The model is two-dimensional where aggregate and mortar are treated as separate constituents having their own characteristic properties. The aggregate is represented as ellipses and generated under prescribed grading curves. Ellipses are randomly placed so it requires probabilistic analysis for model using the Monte Carlo simulation with 20 realizations to represent geometry uncertainty. The nonlinear behavior is simulated with an isotropic damage model for the mortar, while the aggregate is assumed to be elastic. The isotropic damage model softening behavior is defined in terms of fracture mechanics parameters. This damage model is compared with the fixed crack model in macroscale study before using it in the mesoscale model. Then, it is used in the mesoscale model to simulate flexure test and compared to experimental data and shows a good agreement. The probabilistic behavior of the model response is presented through the standard deviation, moment parameters and cumulative probability density functions in different loading stages. It shows variation of the probabilistic characteristics between pre-peak and post-peak behaviour of load-CMOD curves.
\end{abstract}

Keywords: concrete beam, flexure, grading curve, mesoscale, probabilistic analysis, uncertainty.

\section{Introduction}

Over the years, numerical modeling of concrete materials has been conducted using several methodologies. Macroscale models are the most common methodology where concrete is modeled as a homogenous material. Two distinct approaches can be used to modelling: continuum models and fiber-based models. Continuum models are the most commonly used approach, that is employed in most commercial finite element software programs, but they are computationally expensive [1-3]. Fiber-based models, on the contrary, are less common and not computationally expensive [4-6]. Recently, another modeling approach has evolved which is the mesoscale modeling.

Mesoscale analysis has been used as an alternative approach for macroscale analysis for the simulation of heterogeneous materials including concrete. It is powerful when more realistic damage prediction and crack growth is required [7]. Mesoscale analysis of concrete involves modeling aggregate and mortar as separate constituents. Sometimes, the interfacial transition zone between the aggregate and mortar is modeled as a third material [8].

The geometry of a heterogeneous-material is modeled using image processing techniques or through random generation of aggregates. Aggregate shapes can either be rounded or have sharp angles. Size distribution, on the other hand, is modeled using prescribed grading curves or emprical equations such as Fuller's curve $[9,10]$. Material models used to represent fracture in materials include contininuum models such as smeared crack models, damage models, and plastic-damage models [8]. Other models include discrete cracks implemented in the rigid-body-spring model [11], or discrete cracks included in finite elements such as extended finite elements [12].

\footnotetext{
${ }^{*}$ To whom correspondence should be addressed
} 
In many studies, mesoscale models were used to simulate specimens subjected to tension [7, 8, 13-16] and compression [14-19]. Some studies utilized a mesoscale analysis to model beams subjected to flexure. However, most of these research works employed rigid-body-spring models for the simulation of beams [11, 20-22] and others used continuum models with damage and damage-plasticity material models [8, 23]. Some of the mesoscale studies investigated the effect of random aggregate distribution and size on response of the materials, but those studies that employed continuous models in flexure did not perform probabilistic analysis for the variation of the results with different realizations.

This paper is focused on the random behaviour of plain concrete beams subjected to flexure where a two-dimensional continuous mesoscale model is used for the analysis. The model includes two materials: aggregate and mortar. The aggregate is assumed to be elastic while the mortar is modelled using an isotropic damage model. The randomness in the model is induced by the random geometry of the aggregate particles. The position and size of aggregate particles are generated by random variables and their diameter follows a presribed grading curve. Before proceeding with the analysis using the isotropic damage model, a verification process was performed to ensure the validity of the model. The validation was performed by employing the proposed isotropic damage model to analyze the macroscale model and by comparing the results with a fixed crack model and also with experimental load-deflection data. The verification study showed that the isotropic damage model is able to simulate concrete softenning behaviour. After that, the model was used to model mortar in the mesoscale study. The results from probabilistic analysis of 20 realizations were compared with experimental data through load-crack mouth opening displacement-(CMOD) curves and crack propagation. The probabilistic characteristics of response are studied through calculating the mean, standard deviation, variation, skewness, kurtosis, and cumulative probability density functions of the load through different loading stages. The effect of the number of realizations on the convergence of results is also investigated.

The paper is organized as follows. After describing material models used in this work in Section 2, the verification study using the macroscale model is presented in Section 3, and followed by the mesoscale analysis in Section 4. The conclusions are finally drawn in Section 5.

\section{Material models}

Material models for fracture mechanics can be divided into two types: constitutive models that represent cracks in terms of a decrease in constitute stiffness such as smeared crack models, damage models and damage-plasticity models. Other models simulate the crack as discontinuity in the displacement field such as embedded finite elements [24] and extended finite elements [12]. The first type of models has been widely implemented because of its compatibility with the finite element analysis [25-27].

In the following subsections, the isotropic damage model and fixed crack model are described. In both models, softening curves are defined as a function of fracture mechanics parameters.

\subsection{Isotropic damage model}

This is the simplest type of damage models. It assumes that the damage in all directions is equal and represented by a scalar value $\omega[28,29]$.

The relationship between stress and strain is defined in the equation:

$$
\sigma=(1-\omega(\epsilon)) D \varepsilon
$$

The damage can be defined as a function of fracture mechanics parameters using the following formula [30]:

$$
\omega(\epsilon)=1-\frac{\epsilon_{0}}{\epsilon} * \exp \left(-\frac{E \epsilon_{0} h}{G_{f}}\left(\epsilon-\epsilon_{0}\right)\right) .
$$


It is important to note that the above equation is valid to model the stress-strain curve with exponential softening. If other softening type is intended, different equations should be used.

Here, $G_{f}$ is the fracture energy and $E$ is the elastic modulus, while $\epsilon_{0}$ is defined as:

$$
\epsilon_{0}=\frac{f_{t}}{E}
$$

here $\epsilon$ is the highest equivalent strain the material ever experienced and related to the equivalent strain, $\varepsilon_{e q}$, in case of loading and unloading using the following inequalities:

$$
\begin{aligned}
& \varepsilon_{e q}>\epsilon \Rightarrow \epsilon=\varepsilon_{e q}(\text { Loading }), \\
& \left.\varepsilon_{e q} \leq \epsilon \Rightarrow \epsilon=\epsilon \text { (Unloading }\right) .
\end{aligned}
$$

The equivalent strain, on the other hand, is defined as:

$$
\varepsilon_{e q}=\sqrt{\varepsilon_{1 b}^{2}+\varepsilon_{2 b}^{2}}
$$

where

$$
\begin{array}{lllll}
\varepsilon_{1 b}=\varepsilon_{1} \quad \text { if } \quad \varepsilon_{1}>0 \quad \text { otherwise } & \varepsilon_{1 b}=0, \\
\varepsilon_{2 b}=\varepsilon_{2} \quad \text { if } \quad \varepsilon_{2}>0 \quad \text { otherwise } & \varepsilon_{2 b}=0
\end{array}
$$

where $\varepsilon_{1}$ and $\varepsilon_{2}$ are the major and minor principal strains, respectively. Here $h$ is the equivalent element size determined from Eq.(2.9) for a quadrilateral element and from Eq.(2.10) for a triangular element.

$$
\begin{aligned}
& h=\sqrt{A_{e}}, \\
& h=\sqrt{2 A_{e}}
\end{aligned}
$$

where $A_{e}$ is the area of the element.

It has been shown that the isotropic damage model has low mesh sensitivity unlike smeared crack models that are discussed later [30].

\subsection{Fixed crack model}

The fixed crack model is a type of smeared crack models, which involve fixed, rotating and multifixed crack models. In all models, a crack is initiated when the maximum principal stress violates the tensile strength and the initial orientation of the crack is normal to the maximum principal strain [31]. The crack is initiated by means of Rankine criterion and its direction is perpendicular to the principal stress. The crack strain is related to stress by softening curve and shear retention factor [32]. In these models, the material is no longer isotropic. The difference between smeared crack model types is that in the rotating crack model the crack direction is updated during loading, while in the fixed model the crack direction does not change once it is initiated, while in the multi-fixed model orthogonal cracks are generated [26]. In the current study, the fixed crack model is implemented. 
Crack direction is the direction of principal stress when it reaches the tensile strength $f_{t}$

$$
\begin{aligned}
& \varepsilon=\varepsilon_{e l}+\varepsilon_{c r}, \\
& \sigma=D \varepsilon_{e l} .
\end{aligned}
$$

Cracking strain is divided into normal cracking strain and shear cracking strain. The relation between normal cracking strain $\varepsilon_{c r, n}$ and normal stress $\sigma_{n}$ at direction $n$ for exponential softening is described by the following formula [33]

$$
\sigma_{n}=f_{t} * \exp \left(-\frac{h^{*} f_{t}^{*} \varepsilon_{c r, n}}{G_{f}}\right)
$$

In the case of unloading and reloading the equation will be:

$$
\sigma_{n}=\frac{\sigma_{c r, n, \max }}{\varepsilon_{c r, n, \max }} \varepsilon_{c r, n}
$$

where $\varepsilon_{c r, n, \max }$ is the maximum normal cracking strain reached, and $\sigma_{c r, n, \max }$ is the corresponding stress. The relationship between shear stress and shear cracking strain in the tangent direction is defined by

$$
\tau_{t}=\frac{\beta}{1-\beta} G_{c} \gamma_{c r, t}
$$

where $G_{c}$ is the shear modulus and $\beta$ is the shear retention factor, which is assumed to be zero in the current study.

The evaluation of the principal stresses requires an iterative solution process using Newton's Raphson method [34]. It is well known that results using smeared crack models are affected by elements type, mesh size and shape. To overcome this problem, non-local smeared crack models were introduced. These models take the average of strain of neighboring elements within the integral radius, which is an additional parameter for the model to be selected [35].

\section{Macroscale analysis: comparisons with Petersson's beams}

Before the mesoscale analysis, material models were employed in the macroscale analysis with two different types of mesh.

Six simply supported plain concrete beams cast from the same material were tested by Petersson [36]; the beams were $200 \mathrm{~mm}$ in depth and $50 \mathrm{~mm}$ in width with a span of $2000 \mathrm{~mm}$ and were subjected to a concentrated load at the mid-span.

Table 1. Material properties of the beams.

\begin{tabular}{cccc}
\hline$E(\mathrm{MPa})$ & $v$ & $f_{t}(\mathrm{MPa})$ & $G_{f}(\mathrm{~N} / \mathrm{mm})$ \\
\hline 30000 & 0.2 & 3.33 & 0.124 \\
\hline
\end{tabular}


The experimental load-deflection was compared to numerical models of the beams with two mesh types; structured and unstructured mesh as shown in Fig.1. The two types of concrete material models explained earlier were used for each mesh pattern. Concrete properties are provided by Petersson [36] including fracture parameters as listed in Tab.1. Figure 2 shows the load-deflection envelope for all experimental beams compared with the four numerical results including two mesh patterns and two types of material models. The selected softening curve for material is exponential. Bilinear softening of Petersson was used and gave similar results but it is not shown in this paper.

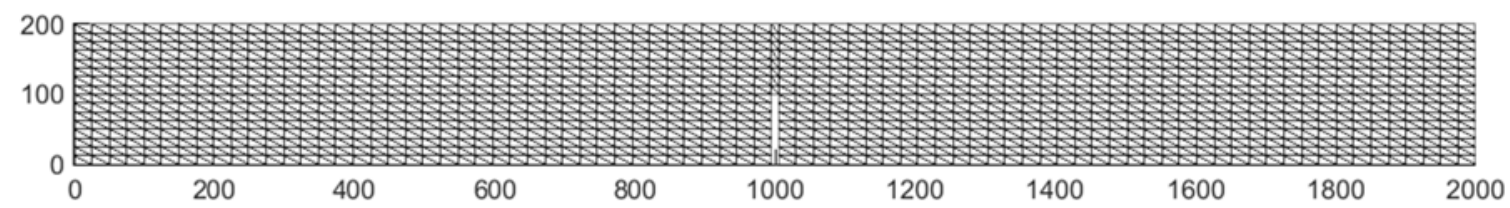

(a) Structured mesh.

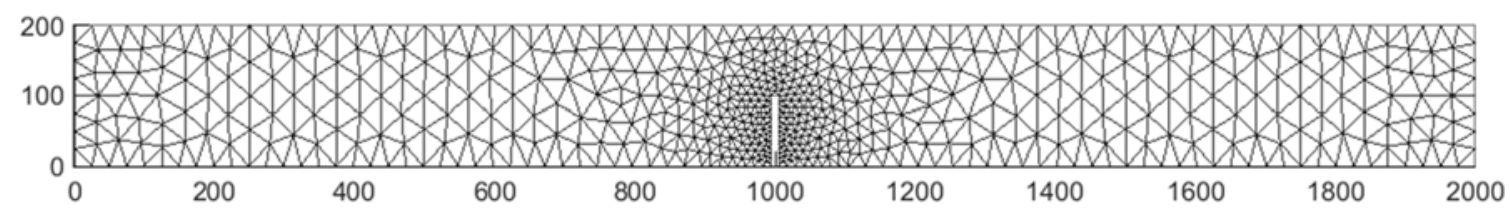

(b) Unstructured mesh.

Fig.1. Structured and unstructured mesh.

It can be seen from Fig.2. that the results obtained with the isotropic damage model agree with those from the experiment. Results of the fixed crack model do not differ much from expeirmental results. Also, it is clear from the figure that the fixed crack model has more mesh sensitivity than the other material model. The stiffer behavior of the fixed crack model is explained by stress locking phenomenon which occurs due to the strain compatibility of the crack and also due to the fact that the stiffness of the normal stress tangent to the crack is not reduced [26]. Figure 3 shows the deflected shape and damage patterns of the beams.

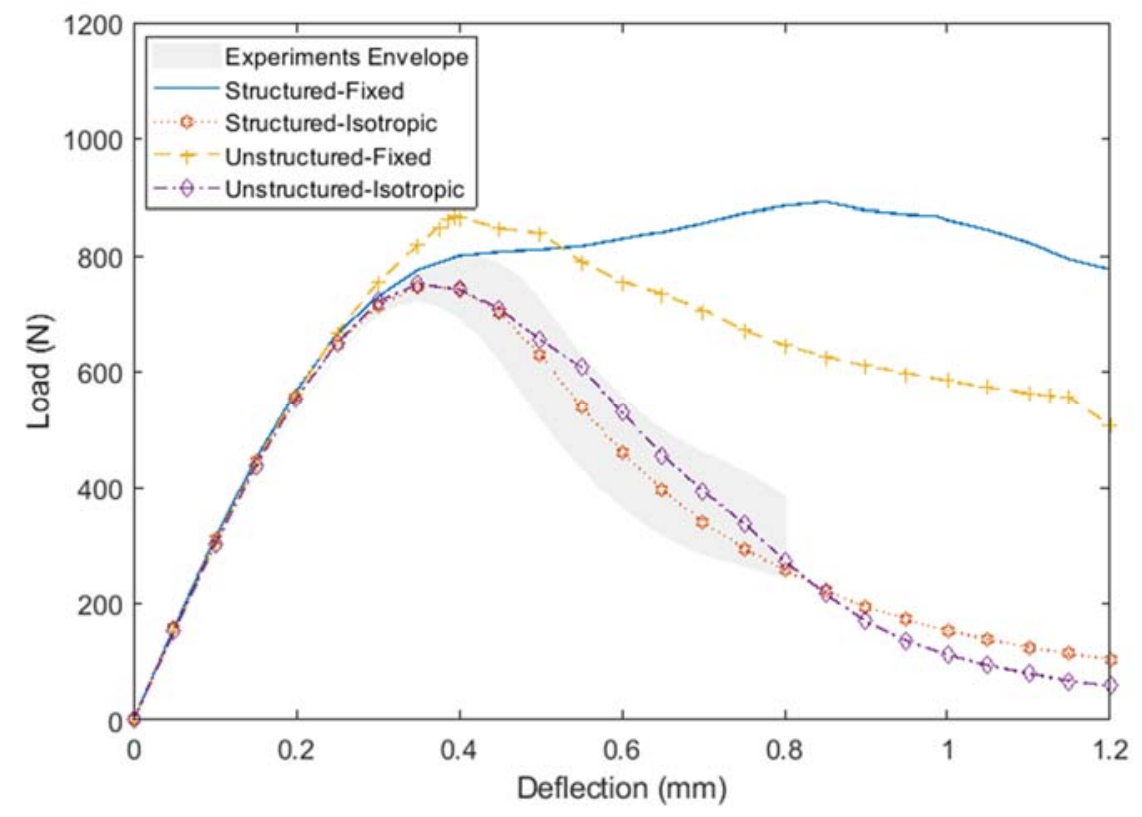

Fig.2. Load-deflection curves of experimental data and numerical models. 


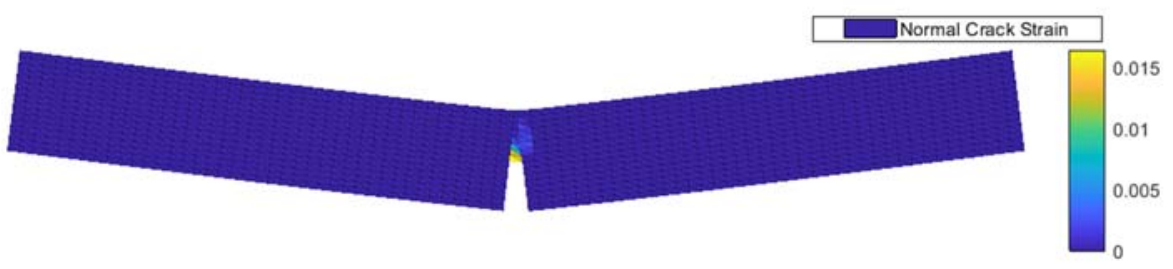

(a) Structured-fixed crack model.

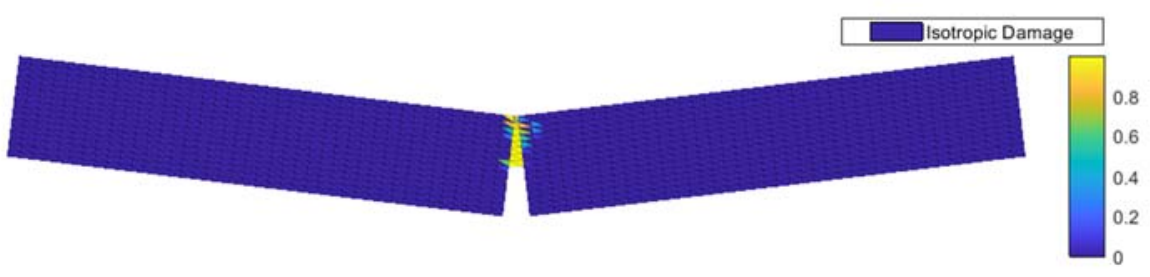

(b) Structured-isotropic damage model.

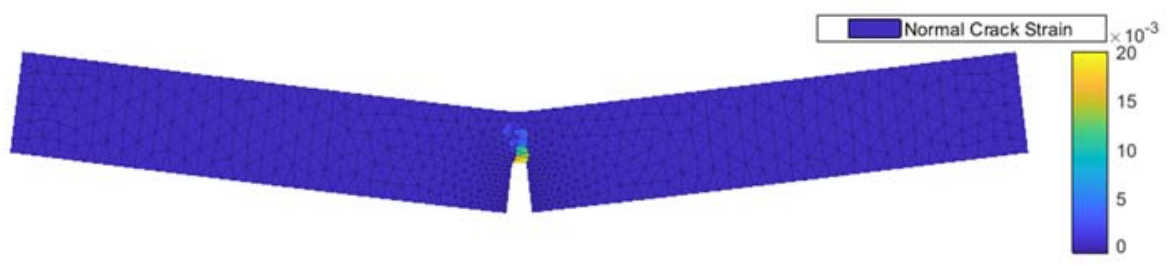

(c) Unstructured-fixed crack model.

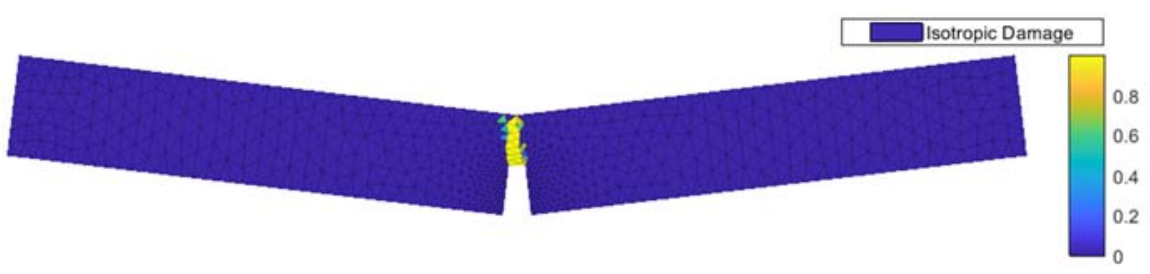

(d) Unstructured-isotropic damage model.

Fig.3. Beams deflected shapes and damage patterns when midspan deflection equals $1.2 \mathrm{~mm}$ (maginification factor is 100 ).

\section{Probabilistic mesoscale analysis: comparision with Grégoire beam}

Grégoire et al. [37] tested beams with different depths and notch heights. All dimensions were scaled according to the beam depth. The out-of-plane thickness was $50 \mathrm{~mm}$. The load and support were applied through bearing plates of width $(0.2 \mathrm{~d})$. Beams had a length of $(3.5 \mathrm{~d})$ and spaned over a distance of $(2.5 \mathrm{~d})$. The selected beam for comparison was $50 \mathrm{~mm}$ in depth with a notch height of $50 \%$ of the beam height. The maximium aggregate size was $10 \mathrm{~mm}$ and the percentage of the aggregate volume from size $5-10 \mathrm{~mm}$ was $30 \%$ of the total speciemen volume. Material properties of these beams were identified by Saliba et al. [38]. In the current study the same material properties identified by Saliba were used except that the aggregate was assumed to be elastic as shown in Tab.2. 
Table 2. Material properties of the beams.

\begin{tabular}{|c|c|c|c|c|}
\hline Material & $E(M P a)$ & $v$ & $f_{t}(M P a)$ & $G_{f}(\mathrm{~N} / \mathrm{mm})$ \\
\hline Mortar & 26 & 0.2 & 3.8 & 0.06 \\
\hline Aggregate & 60 & 0.2 & - & - \\
\hline
\end{tabular}

In the following subsections, the results of generated mesoscale models are compared with the experimental data. First, the methodolgy of aggregate geometry formation as well as mesh generation is presented in Section 4.1; while Section 4.2 includes the probabilistic nonliner analysis of the model. Section 4.3 discusses the effect of loading stages and number of realizations on the results.

\subsection{Geometry and mesh generation}

To get a realistic representation of the mesoscale structure of concrete, shape and size distribution of the aggregate were taken into account using a grading curve. The grading curves can be obtained using criteria of empirical equations available in the related references such as Fuller's curve. In the current study, Fuller's curve, which is expressed in the following form, was utilized for aggregate formation:

$$
\operatorname{Pas} \sin g(\%)=\left(\frac{d}{d_{\max }}\right)^{a} \times 100
$$

where $d$ is the sieve size and $d_{\max }$ is the maximum diameter of aggregate. Passing (\%) is the passing percentage of the $d$ sieve size and $\alpha$ is Fuller's curve exponent and assumed to equal 0.5 in the current study (Fig.4.a.).

After that, ellipsoids were generated in three-dimensional domain. The dimensions of each individual ellipsoid were determined from the sieve curve and aspect ratio of the aggregate required. Ellipsoids radii are determined using the following random variable equations [8]:

$$
\begin{aligned}
& d_{\text {eqv }}=2 * r_{2}=\frac{d_{\mathrm{i}} d_{i+1}}{\sqrt{u_{2} d_{i}^{3}+\left(1-u_{2}\right) d_{i+1}^{3}}}, \\
& r_{1}=\left(1+u_{1} * \frac{m-1}{m+1}\right) * r_{2}, \\
& r_{3}=\left(1-u_{3} * \frac{m-1}{m+1}\right) * r_{2}
\end{aligned}
$$

where $d_{e q v}$ is the medium diameter which is chosen from the grading curve after approximating that curve to multilines. $d_{\mathrm{i}}$ and $d_{i+1}$ are the diameter range for which $d_{e q v}$ is chosen. The number of ellipsoids placed for each diameter range is determined from the requirements set by Eq.(4.1). $r_{1}, r_{2}$, and $r_{3}$ are principal radii with $r_{1} \geq r_{2} \geq r_{3} . u_{1}, u_{2}$, and $u_{3}$ are realizations of uniform random variables ranging from 0 to 1 . Here $m$ is a parameter for flatness. If $m=1$ the particles are sphere, and if the value is more than 1 the flatness increases.

Aggregates were placed according to the algorithm explained by Unger et al. [8], where the particles are divided into sets and then placed individually, as shown in Fig.4b. The particles centers are determined 
from the realizations of uniform random variables ranging within the required domain. If the placed particle intersects with another particle, it will be assigned another location.

In order to work with two dimensional problems, the ellipsoids were sliced by a plane (Fig.5a.) to get two dimensional elllipses. MESH2D MATLAB code was implemented to generate triangular mesh for twodimensional geometries [39, 40]. Figure 5b. presents the generated mesh.

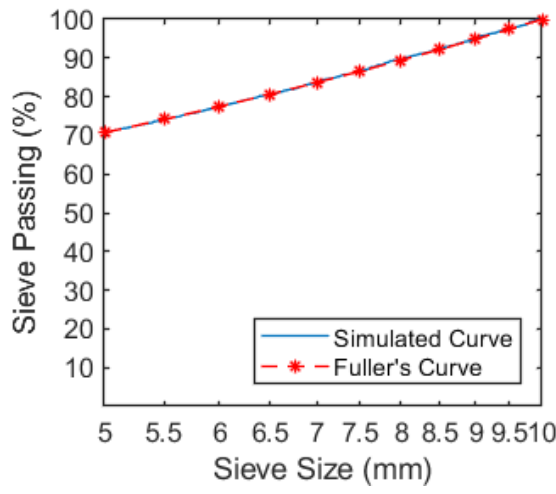

(a) Sieve curve

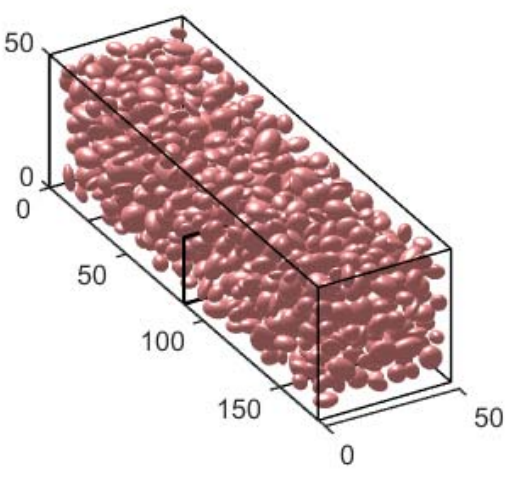

(b) Ellipsoids distribution

Fig.4. Sieve anlysis and ellipsoids distributions.

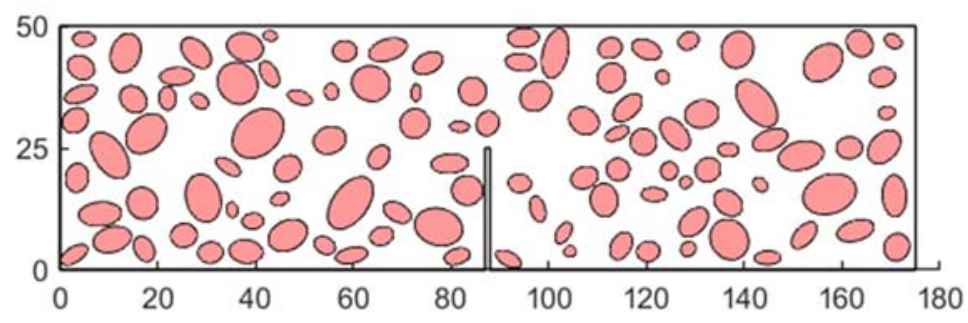

(a) Ellipses.

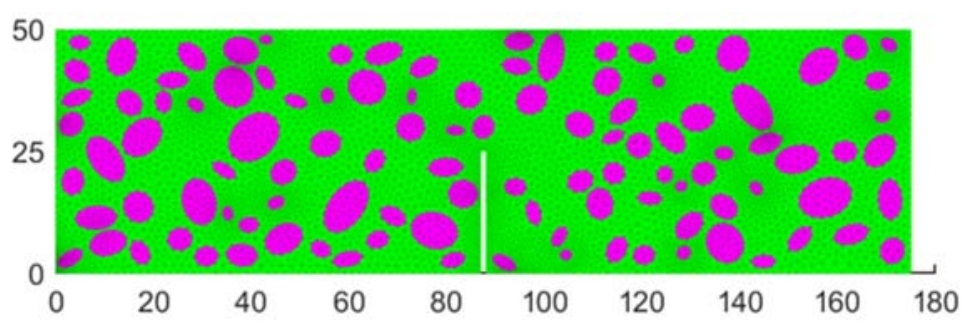

(b) Generated mesh.

Fig.5. Ellipses and generated mesh.

\subsection{Probabilistic mesoscale analysis results}

Since the generated geometry and mesh change with every realization, it is important to perform the analysis with many realizations using the Monte Carlo simulation [41] to get many possible results for aggregate distribution.

Twenty realizations were analyzed. The exponential softening curve was used for concrete softening. Figure 6a shows the load-CMOD curves of the beam for the experimental data along with 20 realizations. The 
mean curve and mean plus/minus one standard deviation curves are also plotted. It can be seen that the numerical model agrees with the experiment near the peak load but underestimes the strengh in the postpeak part. The 20 realizations show similar results with each other. Realization 3 has the lowest peak of load $949.7 \mathrm{~N}$ whereas realization 20 has the highest peak of $1032.3 \mathrm{~N}$. In another study, using the rigid-body-spring model, that was compared with the same experiment, the analysis overestimated the peaks observed in the experiment but showed similar postpeak behavior [21]. In a similar manner, another study, which also used the isotropic damage model, the mesoscale model exhibited a faster decline in strength in the postpeak part compared with the experimental data [23].

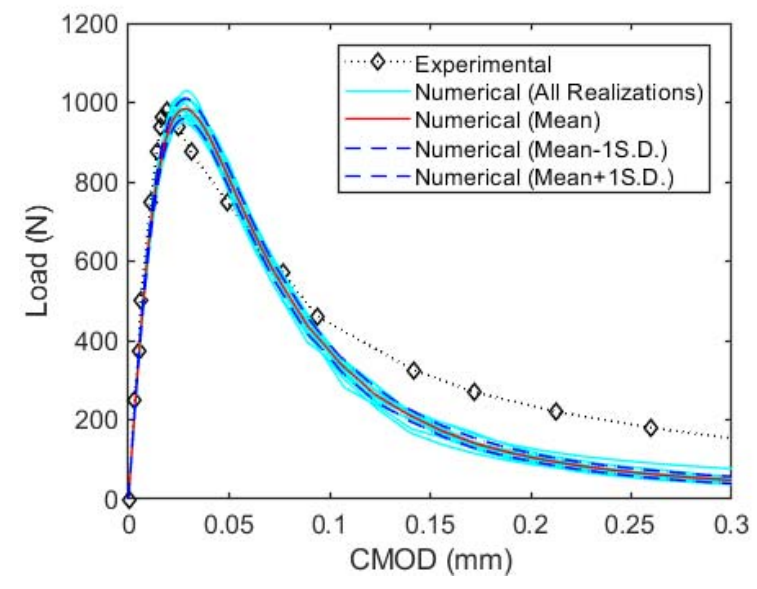

(a) Load-CMOD curves

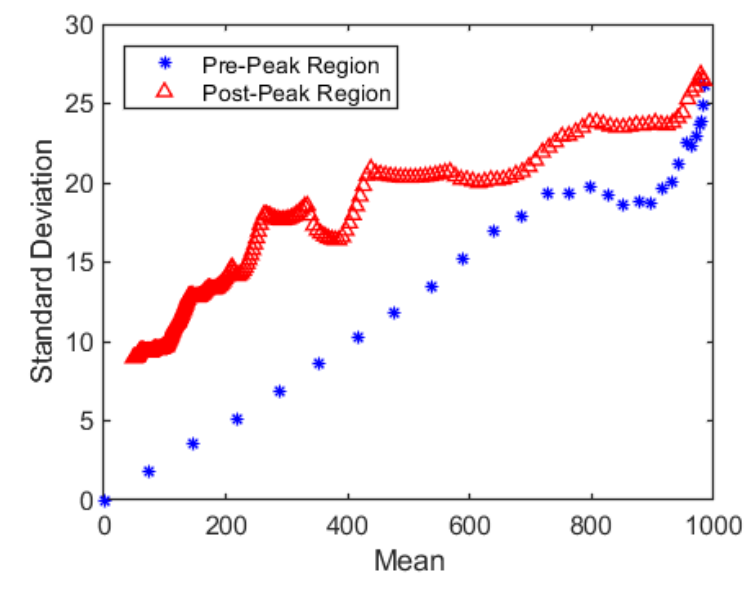

(b) Mean vs. standard deviation of numerical results Fig.6. Load-CMOD curves of experimental data and numerical models.

In Fig.6b. the relationship between the load and its variation of numerical results is studied through plotting the mean with standard deviation. A distinction is made for the data in pre-peak and post-peak regions of the Load-CMOD curves. For the pre-peak region the standard deviation increases with the mean and it is directly proportional at load less than $800 \mathrm{~N}$ in the elastic range. In the post-peak region the standard deviation also increases with the mean but it does not increase linearly with it. Also, it can be seen that the standard deviation is higher for the post-peak than pre-peak region given the same mean value.

Figures 7-8 show the crack growth for different stages of loading for realization 3 and 20. Crack propagation in realization 20 developed two cracks which could be the reason behind the higher peak.

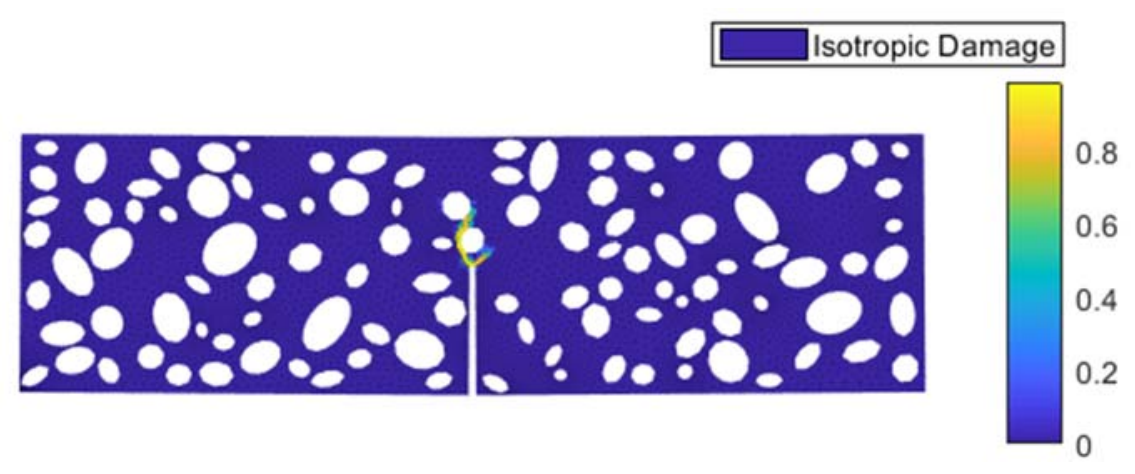

Fig.7a. Crack propagation of realization 3 (maginification factor is 20): $C M O D=0.02545 \mathrm{~mm}$. 


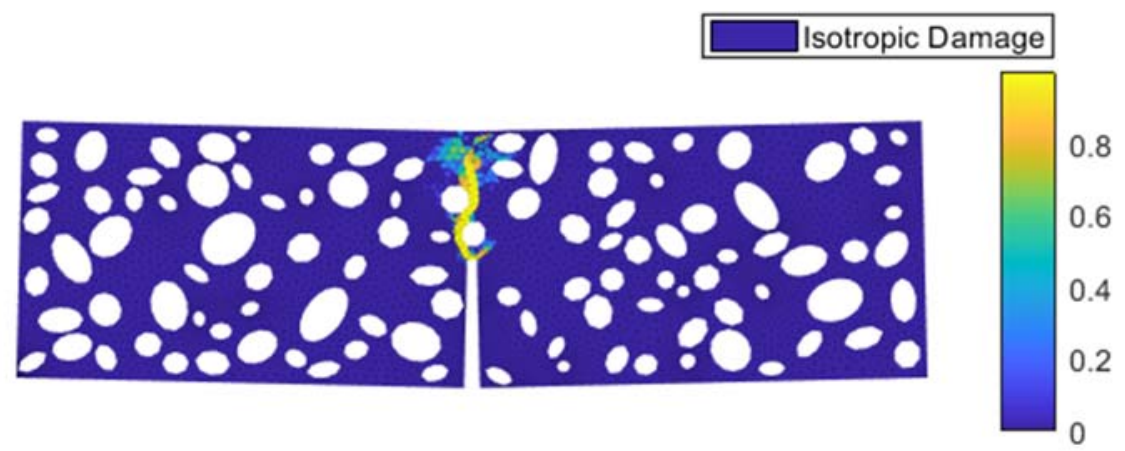

Fig.7b. Crack propagation of realization 3 (maginification factor is 20): $C M O D=0.1047 \mathrm{~mm}$.

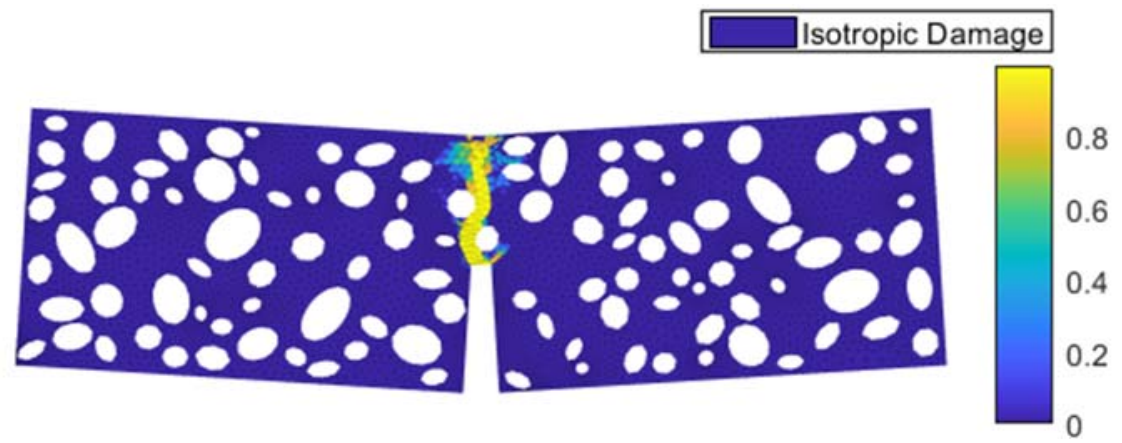

Fig.7c. Crack propagation of realization 3 (maginification factor is 20): $C M O D=0.3021 \mathrm{~mm}$.

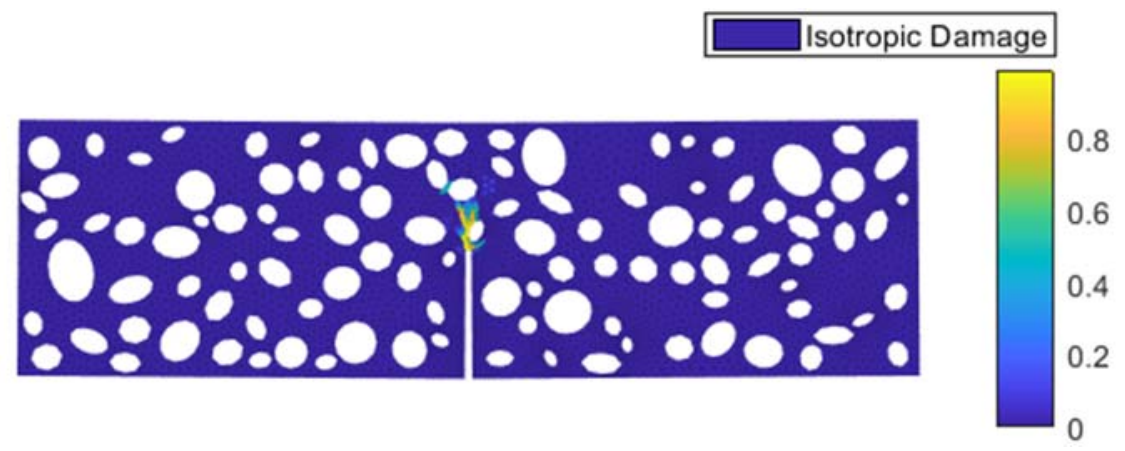

Fig.8a. Crack propagation of realization 20 (maginification factor is 20): $C M O D=0.02558 \mathrm{~mm}$.

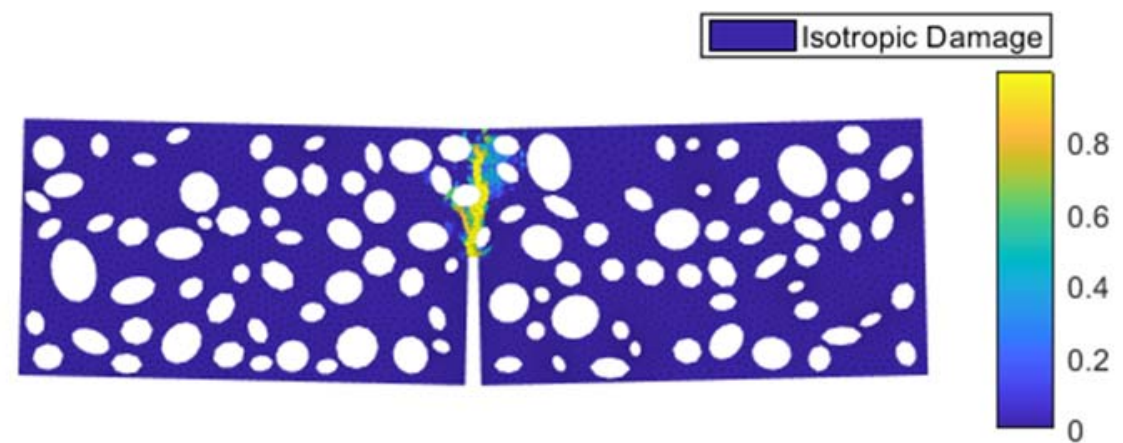

Fig. 8 b. Crack propagation of realization 20 (maginification factor is 20 ): $C M O D=0.1064 \mathrm{~mm}$. 


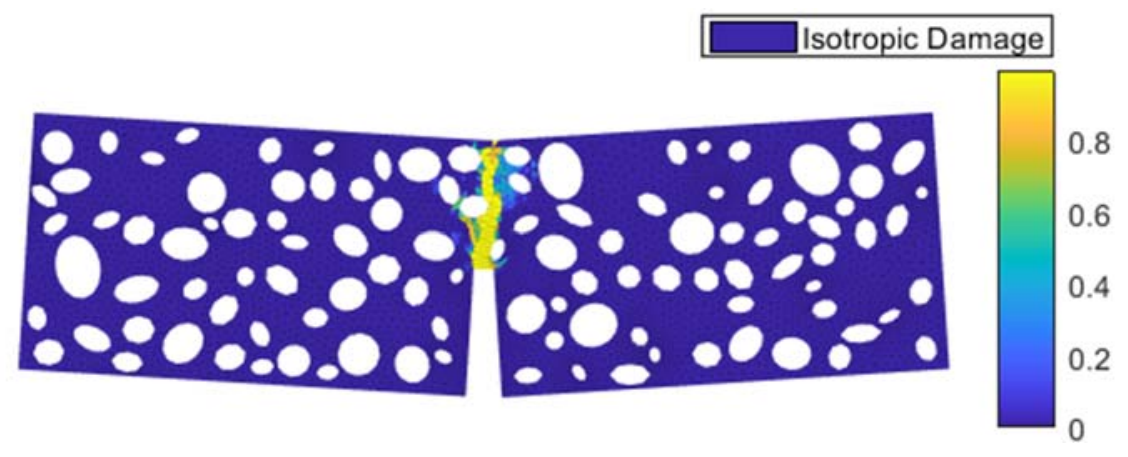

Fig.8c. Crack propagation of realization 20 (maginification factor is 20 ): $C M O D=0.3032 \mathrm{~mm}$.

\subsection{Effect of loading stages and the number of realizations}

The effect of loading stages and the number of realizations on the the load distributions is studied by calculating the mean, variance, skewness and kurtosis for five CMOD values using increasing number of realizations.

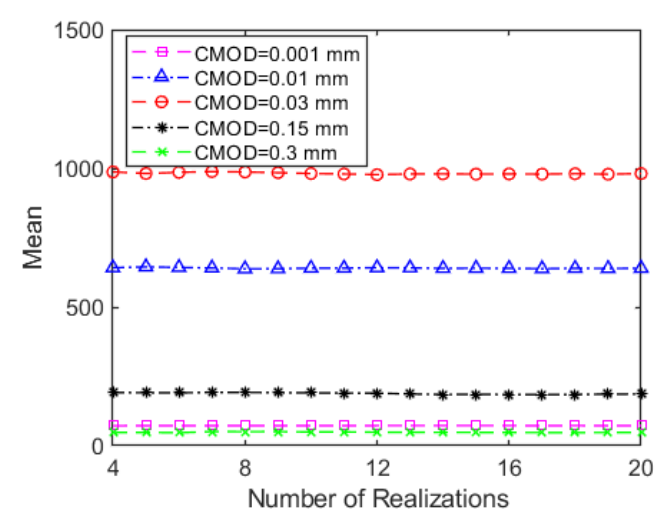

(a) Mean

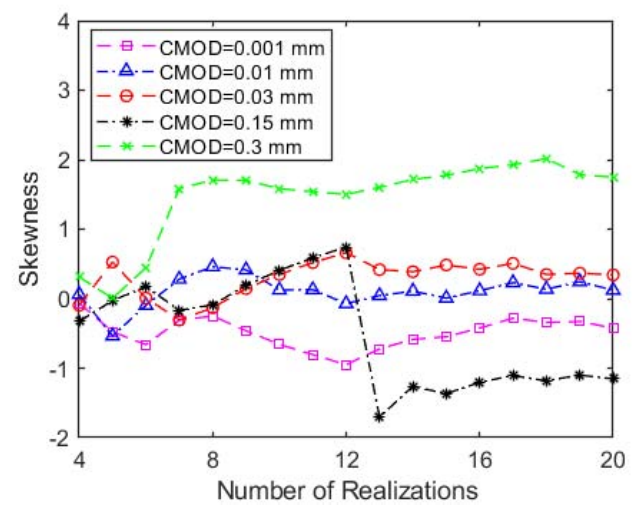

(c) Skewness

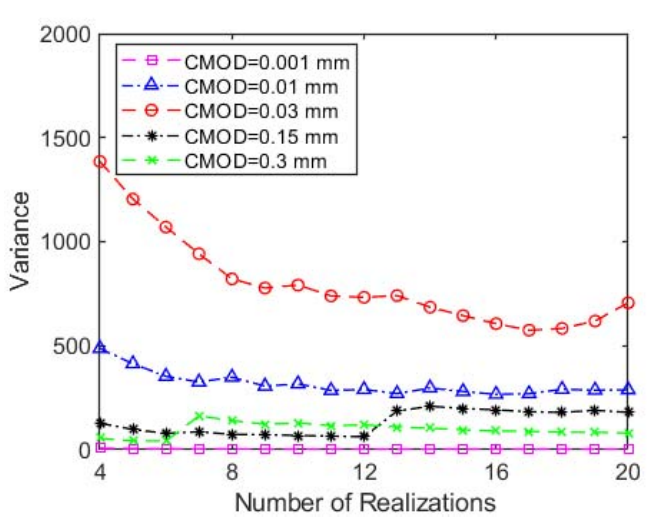

(b) Variance

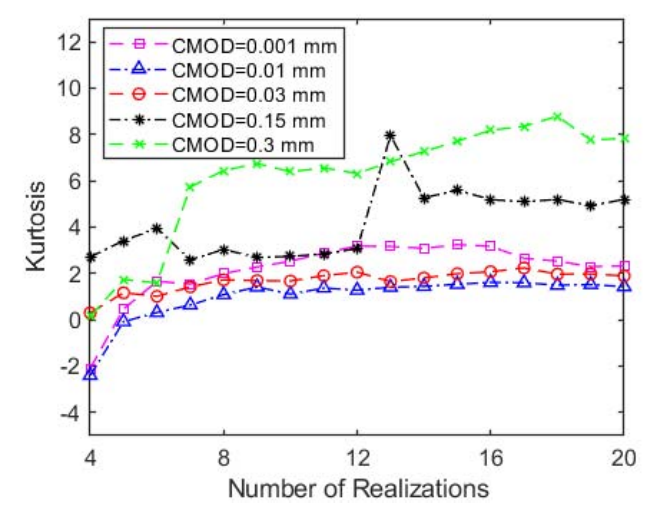

(d) Kurtosis

Fig.9. Variation of mean, variance, skewness and kurtosis curves with the number of realizations for different CMOD values. 
These fours parameters correspond to the first four moments of a random variable. Figure 9 shows the value of these parameters at CMOD values of $0.001 \mathrm{~mm}$ and $0.01 \mathrm{~mm}$ for the pre-peak region, $0.03 \mathrm{~mm}$ near the peak, and values of $0.15 \mathrm{~mm}$ and $0.3 \mathrm{~mm}$ for the post-peak region. The number of realizations ranges from 4 to 20; 4 is used because it is the minimium number of samples needed to calculate kurtosis. The values of the mean are stable with various realizations, while variance has more fluctuations. Skewness and kurtosis show more deviation especially for post-peak values of $0.15 \mathrm{~mm}$ and $0.3 \mathrm{~mm}$. However, their values seem to be around the values of the uniform random variables of 0 and 1.8 in the pre-peak region. The reason behind that could be that the geometry is generated using unifrom random variables. The behaviour should follow more normal distribution after the crack initiation in the post-peak region because of the compound effect of multiple factors. In order to get more precise results, more realizations which are more computationally expensive, are needed for all values to converge.

Figure 10 shows the variation of the cumulative probabiliy distribution function (CDF) for different CMOD values using all 20 realizations and drawn with uniform distribution. The loads are linearly normalized from 0 to 1 to include all curves in one diagram. Similar to the previous observations, CDF curves of CMOD values of $0.001 \mathrm{~mm} ; 0.01 \mathrm{~mm}$, and $0.03 \mathrm{~mm}$ are close to CDF values of the uniform random variable. More realizations are required for more accurate curves, especially for the post-peak region.

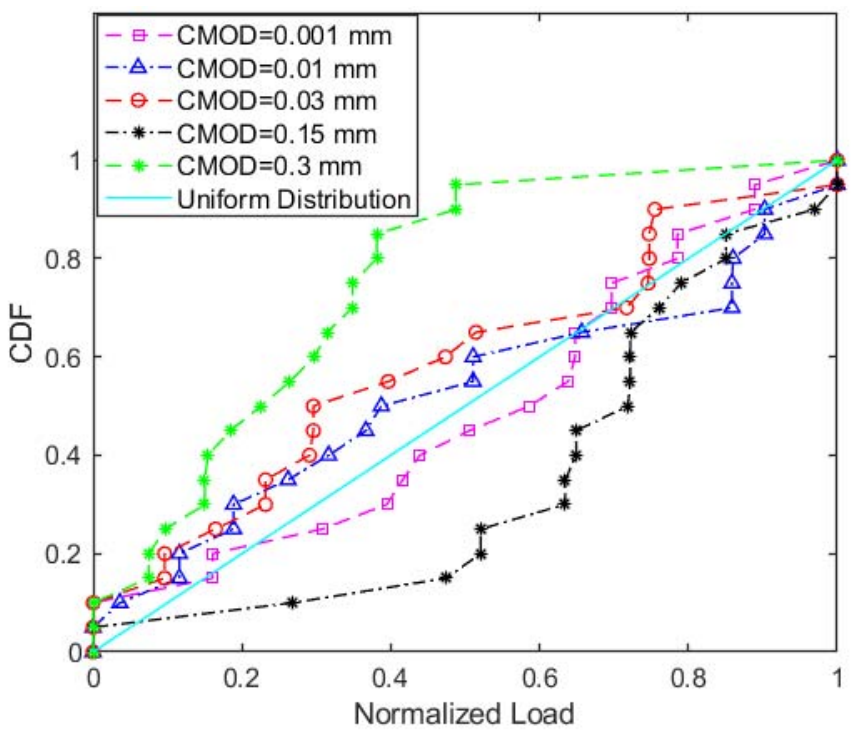

Fig.10. The variation of CDF for different CMOD values using 20 realizations.

\section{Conclusions}

In the present study, the probabilistic behavior of plain concrete beams subjected to flexure was investigated using the two-dimensional continuous mesoscale model. The study involved examining material models in macroscale model before proceeding to mesoscale analysis. The comparison revealed that the isotropic damage model results agreed with the experiemental results while the fixed crack model showed overstiff behavior for different mesh patterns. Then, the isotropic damage model was utilized in the mesoscale model with 20 realizations to simulate a beam subjected to flexure and compared with the experimental loadCMOD curves. Load-CMOD curves for both models showed good aggrement. Furthermore, the crack growth presentation was more realistic than the ones obtained from the macroscale models. The probabilistic analysis of the results showed different patterns of behaviour in the pre-peak and post-peak regions. The standard deviation values of the loads were correlated to the mean load and were directly proportional in the pre-peak region. However, they were higher for the post-peak region. The distributions characteristics are studied through mean, variation, skewness, kurtosis and and cumulative probability density functions for different 
CMOD values and number of realizations. The comparison showed that load probability distributions are similar to uniform random variables in the pre-peak region, and had fluctuations in the post-peak region. Increasing the number of realizations revealed that the mean values converged, while exhibited slower convergence. Skewness and kurtosis apeared to be more swinging, especially for CMOD values in the postpeak region. More realizations are needed for all values and distributions to converge, especially for the postpeak region where the model shows a more complex nonlinear behaviour.

\section{Nomenclature}

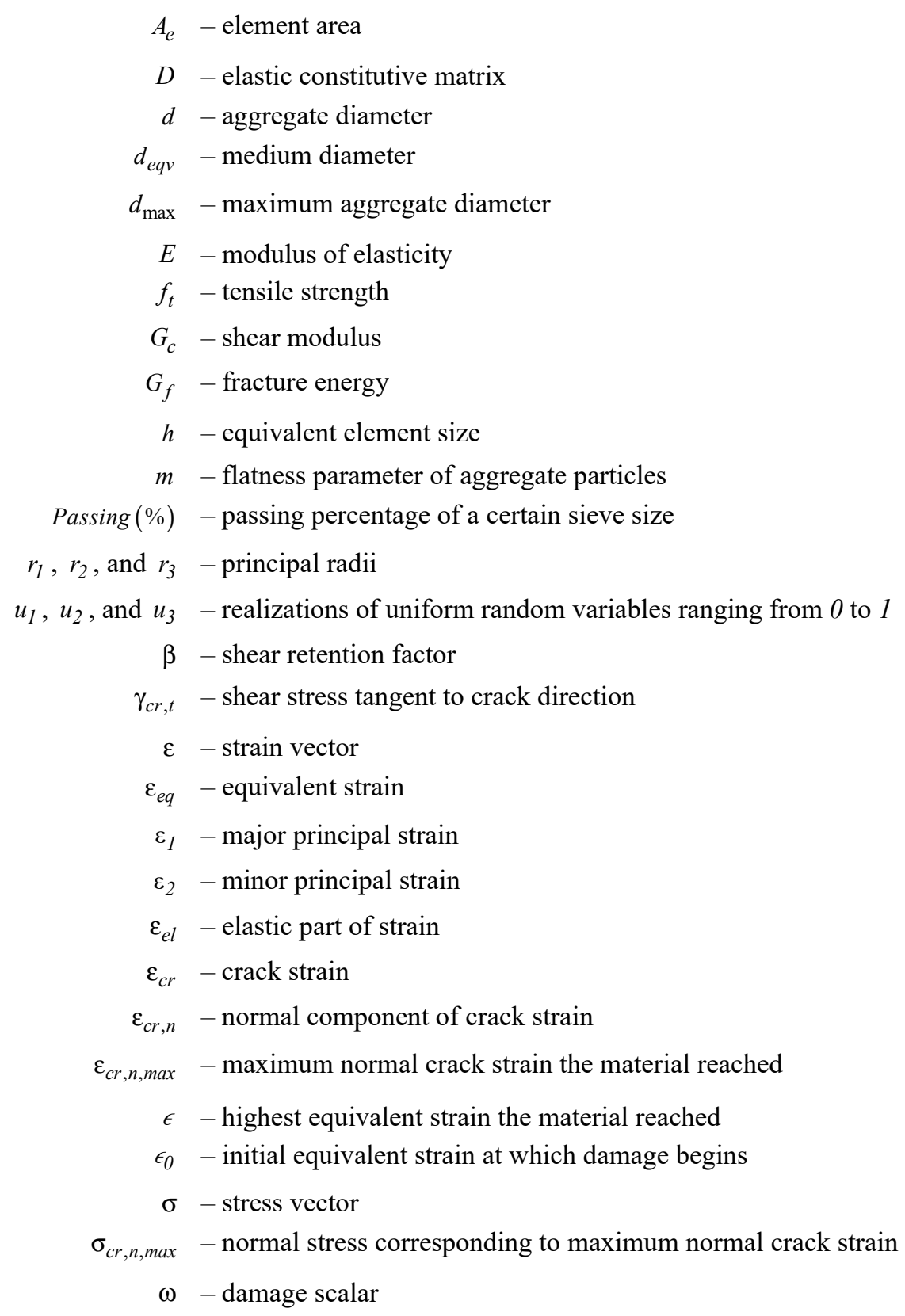

\section{Conflict of Interest Statement}

On behalf of all authors, the corresponding author states that there is no conflict of interest. 


\section{Funding}

No funding was received for conducting this study.

\section{Author Contributions}

All authors whose names appear on the submission made substantial contributions that included, but not limited to, the numerical program written and used in the work. Also, they all contributed in data interpretation and manuscript preparation.

\section{References}

[1] Kachanov M. (1980): Continuum model of medium with cracks.- Journal of the engineering Mechanics Division, vol.106, No.3, pp.1039-1051.

[2] Belletti B., Cerioni R. and Iori I. (2001): Physical approach for reinforced-concrete (PARC) membrane elements.Journal of Structural Engineering, vol.127, No.12, pp.1412-1426.

[3] Menin R. C.G., Trautwein L.M. and Bittencourt T.N. (2009): Smeared crack models for reinforced concrete beams by finite element method.- IBRACON Structures and Materials Journal, vol.2, No.2, pp.166-200.

[4] Al-Jelawy H.M., Mackie K.R. and Haber Z.B. (2018): Shifted plastic hinging for grouted sleeve column connections.ACI Structural Journal, vol.115, No.4, pp.1101-1114.

[5] Al-Jelawy H.M., Mackie K.R. and Haber Z.B. (2018): Experimental and numerical studies on precast bridge columns with shifted plastic hinging.- Eleventh US National Conference on Earthquake Engineering, pp.25-29.

[6] Haber Z.B., Mackie K.R. and Al-Jelawy H.M. (2017): Testing and analysis of precast columns with grouted sleeve connections and shifted plastic hinging.- Journal of Bridge Engineering, vol.22, No.10, pp.04017078.

[7] Unger J.F., Eckardt S. and Kooenke C. (2017): A mesoscale model for concrete to simulate mechanical failure.Computers \& Concrete, vol.8, No.4, pp.401-423.

[8] Unger J.F. and Eckardt S. (2011): Multiscale modeling of concrete.- Archives of Computational Methods in Engineering, vol.18, No.3, pp.341-393.

[9] Häfner S., Eckardt S., Luther T. and Könke C. (2006): Mesoscale modeling of concrete: Geometry and numerics.Computers \& structures, vol.84, No.7, pp.450-461.

[10] Zhang Z., Song X., Liu Y., Wu D. and Song C. (2017): Three-dimensional mesoscale modelling of concrete composites by using random walking algorithm.- Composites Science and Technology, vol.149, pp.235-245.

[11] Grassl P. and Bažant Z.P. (2009): Random lattice-particle simulation of statistical size effect in quasi-brittle structures failing at crack initiation.- Journal of Engineering Mechanics, vol.135, No.2, pp.85-92.

[12] Moës N., Dolbow J. and Belytschko T. (1999): A finite element method for crack growth without remeshing.International Journal for Numerical Methods in Engineering, vol.46, No.1, pp.131-150.

[13] Kim S.M. and Al-Rub R.K. (2011): Meso-scale computational modeling of the plastic-damage response of cementitious composites.- Cement and Concrete Research, vol.41, No.3, pp.339-358.

[14] Chen H., Xu B., Mo Y.L. and Zhou T. (2018): Behavior of meso-scale heterogeneous concrete under uniaxial tensile and compressive loadings.-Construction and Building Materials, vol.178, pp.418-431.

[15] Karavelić E., Nikolić M., Ibrahimbegovic A. and Kurtović A. (2019): Concrete meso-scale model with full set of 3D failure modes with random distribution of aggregate and cement phase. Part I: Formulation and numerical implementation.- Computer Methods in Applied Mechanics and Engineering, vol.344, pp.1051-1072.

[16] Zhou R. and Lu Y. (2018): A mesoscale interface approach to modelling fractures in concrete for material investigation.-Construction and Building Materials, vol.165, pp.608-620. 
[17] Niknezhad D., Raghavan B., Bernard F. and Kamali-Bernard S. (2015): The influence of aggregate shape, volume fraction and segregation on the performance of Self-Compacting Concrete: $3 D$ modeling and simulation.Rencontres Universitaires de Genie Civil.

[18] Niknezhad D., Raghavan B., Bernard F. and Kamali-Bernard S. (2015): Towards a realistic morphological model for the meso-scale mechanical and transport behavior of cementitious composites.- Composites Part B: Engineering, vol.81, pp.72-83.

[19] Xie Z.H., Guo Y.C., Yuan Q.Z. and Huang P.Y. (2015): Mesoscopic numerical computation of compressive strength and damage mechanism of rubber concrete.- Advances in Materials Science and Engineering, vol.2015, Article ID 279584, p.10, http://dx.doi.org/10.1155/2015/279584.

[20] Eliáš J., Vořechovský M., Skoček J. and Bažant Z.P. (2015): Stochastic discrete meso-scale simulations of concrete fracture: Comparison to experimental data.- Engineering fracture mechanics, vol.135, pp.1-6.

[21] Grassl P., Grégoire D., Solano L.R. and Pijaudier-Cabot G. (2012): Meso-scale modelling of the size effect on the fracture process zone of concrete.- International Journal of Solids and Structures, vol.49, No.13, pp.1818-1827.

[22] Wang L.C. (2013): Meso-scale numerical modeling of the mechanical behavior of reinforced concrete members.International Journal of Engineering and Technology, vol.5, No.6, pp.680.

[23] Skarżyński Ł. and Tejchman J. (2012): Numerical mesoscopic analysis of fracture in fine-grained concrete.Archives of Civil Engineering, vol.58, No.3, pp.331-361.

[24] Jirásek M. (2000): Comparative study on finite elements with embedded discontinuities.- Computer Methods in Applied Mechanics and Engineering, vol.188, No.1-3, pp.307-330.

[25] Rots J.G. (1988): Computational modeling of concrete fracture.- Delft: Technische Hogeschool Delft.

[26] Rots J.G. and Blaauwendraad J. (1989): Crack models for concrete, discrete or smeared? Fixed, multi-directional or rotating?.- HERON, vol.34, No.1.

[27] Rots J.G., Nauta P., Kuster G.M. and Blaauwendraad J. (1985): Smeared Crack Approach and Fracture Localization in Concrete.- HERON, vol.30, No.1.

[28] Kachanov L. (1986): Introduction to Continuum Damage Mechanics.- Springer Science \& Business Media.

[29] Lemaitre J. and Chaboche J.L. (1994): Mechanics of Solid Materials.- Cambridge University Press.

[30] Kurumatani M., Terada K., Kato J., Kyoya T. and Kashiyama K. (2016): An isotropic damage model based on fracture mechanics for concrete.- Engineering Fracture Mechanics, vol.155, pp.49-66.

[31] Mahmoud K.S., Al-Sherrawi M.H. (2002): Nonlinear finite element analysis of composite concrete beams.- Journal of Engineering, Baghdad, Iraq, vol.3, No.8, pp.273-288.

[32] Rashid Y.R. (1968): Ultimate strength analysis of prestressed concrete pressure vessels.- Nuclear Engineering and Design, vol.7, No.4, pp.334-344.

[33] Eckardt S., Häfner S. and Könke C. (2004): Simulation of the fracture behaviour of concrete using continuum damage models at the mesoscale.- Proceedings of ECCOMAS.

[34] Sena-Cruz J., Barros J.A. and Azevedo Á.F. (2004): Elasto-Plastic Multi-Fixed Smeared Crack Model for Concrete.Universidade do Minho, Departamento de Engenharia Civil (DEC).

[35] Bažant Z.P. and Lin F.B. (1988): Nonlocal smeared cracking model for concrete fracture.- Journal of structural engineering, vol.114, No.11, pp.2493-2510.

[36] Petersson P.E. (1981): Crack Growth And Development Of Fracture Zones In Plain Concrete And Similar Materials.- Lund, Sweden: Lund Institute of Technology.

[37] Grégoire D., Rojas-Solano L.B. and Pijaudier-Cabot G. (2013): Failure and size effect for notched and unnotched concrete beams.- International Journal for Numerical and Analytical Methods in Geomechanics, vol.37, No.10, pp.1434-1452. 
[38] Saliba J., Matallah M., Loukili A., Regoin J.P., Grégoire D., Verdon L. and Pijaudier-Cabot G. (2016): Experimental and numerical analysis of crack evolution in concrete through acoustic emission technique and mesoscale modelling.- Engineering Fracture Mechanics. vol.167, pp.123-137.

[39] Engwirda D. (2014): Locally-optimal Delaunay-refinement and optimisation-based mesh generation.- School of Mathematics and Statistics, The University of Sydney.

[40] Engwirda D. (2005): Unstructured mesh methods for the Navier-Stokes equations.- School of Aerospace, Mechanical and Mechatronic Engineering, The University of Sydney.

[41] Stefanou G. (2009): The stochastic finite element method: past, present and future.- Computer methods in applied mechanics and engineering, vol.198, No.9-12, pp.1031-1051.

Received: January 17, 2021

Revised: April 14, 2021 